Pacific Journal of Mathematics

SEMIGROUPS GENERATED BY CERTAIN OPERATORS ON
VARIETIES OF COMPLETELY REGULAR SEMIGROUPS 


\title{
SEMIGROUPS GENERATED BY CERTAIN OPERATORS ON VARIETIES OF COMPLETELY REGULAR SEMIGROUPS
}

\author{
Mario Petrich and Norman R. ReIlly
}

\begin{abstract}
The operators $C, K, L, T, T_{l}$ and $T_{r}$ on the lattice $\mathscr{L}(\mathscr{C} \mathscr{R})$ of varieties of completely regular semigroups have played an important role in recent studies of $\mathscr{L}(\mathscr{C} \mathscr{R})$. Although each of these operators is idempotent, when applied in various combinations to the trivial variety they yield varieties for which the only upper bound is $\mathscr{C} \mathscr{R}$. The semigroups generated by various subsets of $\left\{C, K, L, T, T_{r}, T_{l}\right\}$ are determined here in terms of generators and relations.
\end{abstract}

1. Introduction and summary. Completely regular semigroups (unions of groups) may be regarded as algebras with the operations of (binary) multiplication and (unary) inversion. As such they form a variety $\mathscr{C} \mathscr{R}$ defined by the identities

$$
(a b) c=(a b) c, \quad a=a a^{-1} a, \quad a a^{-1}=a^{-1} a, \quad\left(a^{-1}\right)^{-1}=a .
$$

The lattice $\mathscr{L}(\mathscr{C} \mathscr{R})$ of all subvarieties of $\mathscr{C} \mathscr{R}$ turns out to be amenable to a thorough analysis both globally and locally. The former includes various (complete) congruences that emerge naturally in the study either of the varieties themselves or of the corresponding fully invariant congruences on a free completely regular semigroup $F \mathscr{C} \mathscr{R}$ on a countably infinite set. Local studies of the lattice $\mathscr{L}(\mathscr{C} \mathscr{R})$ usually amount to rather complete descriptions of relatively small intervals in $\mathscr{L}(\mathscr{C} \mathscr{R})$ modulo $\mathscr{L}(\mathscr{G})$, the lattice of group varieties, starting from the bottom of the lattice.

In the local approach, a number of operators make their appearance in the description of certain varieties in terms of some of their proper subvarieties. But these operators may be defined on all of $\mathscr{L}(\mathscr{C} \mathscr{R})$ thereby providing a certain amount of information for varieties scattered throughout $\mathscr{L}(\mathscr{C} \mathscr{R})$ and hence may be used for a global study of this lattice. Another source of operators on $\mathscr{L}(\mathscr{C} \mathscr{R})$ are the kernel and trace relations on the lattice of fully invariant congruences on $F \mathscr{C} \mathscr{R}$ now translated into relations on $\mathscr{L}(\mathscr{C} \mathscr{R})$.

Of the considerable literature on varieties of completely regular semigroups, we mention only the following ones because they are directly related to our object of study. We thus cite Jones [6], [7], Kadourek [8], 
Pastijn-Trotter [10] and Reilly [13] for various results concerning the operators under study.

In order to explain briefly what the subject of the paper is, we need some notation. For any $S \in \mathscr{C} \mathscr{R}$, let $E(S)$ be the set of idempotents of $S$ and $C(S)$ be the core of $S$, that is the subsemigroup of $S$ generated by $E(S)$. We define operators $C$ and $L$ on $\mathscr{L}(\mathscr{C} \mathscr{R})$ by

$$
\begin{aligned}
& \mathscr{V} C=\{S \in \mathscr{C} \mathscr{R} \mid C(S) \in \mathscr{V}\}, \\
& \mathscr{V} L=\{S \in \mathscr{C} \mathscr{R} \mid e S e \in \mathscr{V} \text { for all } e \in E(S)\} .
\end{aligned}
$$

Note that $\mathscr{V} C$ (respectively $\mathscr{V} L$ ) consists of all $S$ in $\mathscr{C} \mathscr{R}$ all of whose idempotent generated subsemigroups (respectively submonoids) are contained in $\mathscr{V}$.

For a fully invariant congruence $\rho$ on $F \mathscr{C} \mathscr{R}$ (see above), let $[\rho]$ be the corresponding variety. Let $\rho_{K}$ (respectively $\rho_{T}, \rho_{T_{l}}, \rho_{T_{r}}$ ) denote the least (automatically fully invariant) congruence on $F \mathscr{C} \mathscr{R}$ with the same kernel (respectively trace, left trace, right trace) as $\rho$. We now define operators $K, T, T_{l}, T_{r}$ on $\mathscr{L}(\mathscr{C} \mathscr{R})$ by the requirement:

$$
[\rho] P=\left[\rho_{P}\right] \quad\left(P \in\left\{K, T, T_{l}, T_{r}\right\}\right) .
$$

These operators admit the following interpretations: for any $\mathscr{V} \in \mathscr{L}(\mathscr{C} \mathscr{R})$, and with $\mathscr{S}$ denoting the variety of semilattices,

$$
\begin{aligned}
& \mathscr{V} K=\{S \in \mathscr{C} \mathscr{R} \mid S / \tau \in \mathscr{V} \vee \mathscr{S}\}, \\
& \mathscr{V} T=\{S \in \mathscr{C} \mathscr{R} \mid S / \mu \in \mathscr{V}\}, \\
& \mathscr{V} T_{l}=\left\{S \in \mathscr{C} \mathscr{R} \mid S / \mathscr{L}^{0} \in \mathscr{V}\right\},
\end{aligned}
$$

in the usual notation with $\mathscr{L}^{0}$ the greatest congruence contained in $\mathscr{L}$ and a symmetric expression for $\mathscr{V} T_{r}$.

The principal results of the paper consist of complete descriptions of semigroups generated by certain subsets of $\left\{C, L, K, T, T_{l}, T_{r}\right\}$. In order to roughly state these descriptions, let $M_{P, Q}$ (respectively $N_{P, Q}$ ) be the monoid generated by $C, L, P$ and $Q$ (respectively $P$ and $Q$ ) where $\{P, Q\}$ is any 2-element subset of $\left\{K, T, T_{l}, T_{r}\right\}$ and $R$ be the monoid generated by $C$ and $L$. We prove that

$$
M_{P, Q} \cong R \times N_{P, Q},
$$

that $N_{P, Q}$ is a free monoid on two idempotent generators (except when $\{P, Q\} \stackrel{=}{=}\left\{T, T_{l}\right\}$ or $\left.\left\{T, T_{r}\right\}\right)$, and give the multiplication table for the 5-element monoid $R$.

Section 2 contains the needed notation. The semigroup generated by $C$ and $L$ is described in $\S 3$. Properties of the operators $K, T, T_{l}$ and $T_{r}$ are 
discussed in $\S 4$. That $C$ (respectively $L$ ) commutes with $K, T, T_{l}$ and $T_{r}$ is proved in $\$ 5$ (respectively $\S 6$ ). The semigroups generated by pairs of these operators are determined in $\$ 7$, whereas the semigroup generated by $C, L, K$ and $T$ is described in $\S 8$. A diagram of values of some of the elements of the semigroups of operators at the trivial variety is presented in $\S 9$.

2. Preliminaries. A semigroup which is a union of groups is said to be a completely regular semigroup. Basic information about such semigroups can be found in Howie [5]. The fundamental structure theorem for completely regular semigroups, due to Clifford, states that a semigroup $S$ is completely regular if and only if $S$ is a semilattice of completely simple semigroups. We will denote this by $S=\bigcup_{\alpha \in Y} S_{\alpha}$, where $Y$ is a semilattice, and refer to the completely simple subsemigroups $S_{\alpha}$ as the components of $S$.

In a completely regular semigroup $S$, we use the following notation. If $x \in S$, then $x^{-1}$ is the inverse of $x$ in the maximal subgroup of $S$ containing $x$. In addition, let $x^{0}=x x^{-1}$. Also, $E(S)$ denotes the set of idempotents of $S$ and Con $S$ denotes the lattice of congruences on $S$.

Certain congruences on a completely regular semigroup are particularly important. Let $\rho \in \operatorname{Con} S, S \in \mathscr{C} \mathscr{R}$. Then $\rho$ is said to be idempotent

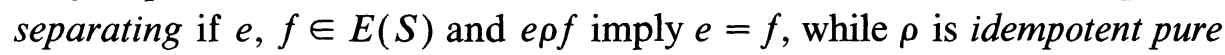
if $e \in E(S)$ and e $a$ imply $a \in E(S)$. We will denote by $\mu=\mu_{S}$ (respectively $\tau=\tau_{S}$ ) the maximum idempotent separating (respectively idempotent pure congruence) on $S$. Also, for any equivalence relation $\lambda$ on $S$, we denote by $\lambda^{0}$ the largest congruence on $S$ contained in $\lambda$. It is sometimes useful to remember that $\mu=\mathscr{H}^{0}$.

The term variety means variety of completely regular semigroups as algebras with multiplication and inversion. We use the following notation for various varieties:

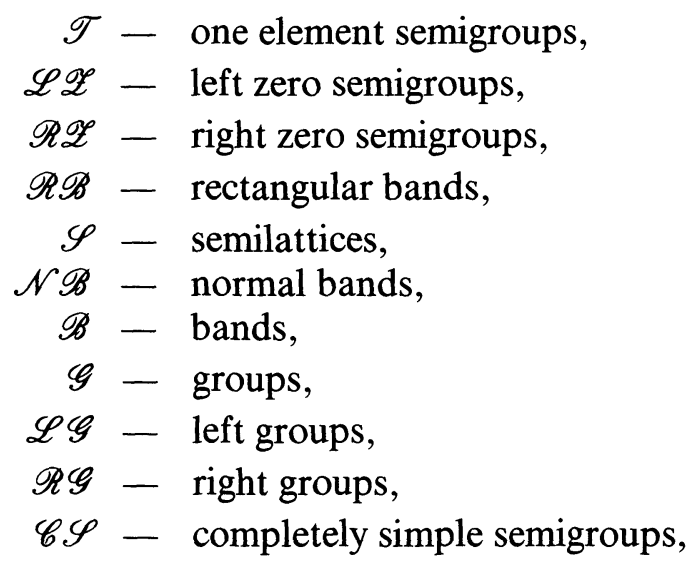




$$
\begin{aligned}
\mathscr{N} \mathscr{B} \mathscr{G} & - \text { normal bands of groups, } \\
\mathscr{R} e \mathscr{G} & - \text { rectangular groups, } \\
\mathcal{O} & - \text { orthodox completely regular semigroups, } \\
\mathscr{C} \mathscr{R} & - \text { completely regular semigroups. }
\end{aligned}
$$

Moreover,

$$
\begin{aligned}
\mathscr{L}(\mathscr{V}) & - \text { the lattice of subvarieties of } \mathscr{V} \\
F \mathscr{V} & - \text { the (relatively) free completely regular semigroup } \\
& \text { on a countably infinite set in a variety } \mathscr{V}, \\
F & =F \mathscr{C} \mathscr{R}, \\
F_{n} & - \text { the free completely regular semigroup on a set of } n \text { elements, } \\
\mathscr{C} & - \text { the lattice of fully invariant congruences on } F .
\end{aligned}
$$

For a set $A$ of operators on $\mathscr{L}(\mathscr{C} \mathscr{R})$, we denote by $[A]$ the subsemigroup of the full transformation semigroup on $\mathscr{L}(\mathscr{C} \mathscr{R})$ generated by $A$. The free semigroup on a nonempty set $X$ is denoted by $X^{+}$. A semigroup given by generators $G$ and relations $R$ is denoted by $\langle G \mid R\rangle$. For a semigroup $S, S^{1}$ (respectively $S^{0}$ ) stands for $S$ with an identity (respectively zero) adjoined. On any set $X, \varepsilon$ denotes the equality relation. Proper inclusion of sets is denoted by $\subset$. The notation $|X|$ stands for the cardinality of a set $X,|w|$ stands for the length of the word $w$, and $|n|$ also denotes the usual absolute value of an integer $n$.

Undefined terminology and notation can be found in [5] and [11].

3. The operators $C$ and $L$. In this section we introduce two operators on $\mathscr{L}(\mathscr{C} \mathscr{R})$ and describe the semigroup that they generate.

For any $S \in \mathscr{L}(\mathscr{C} \mathscr{R})$, let $C(S)$ denote the subsemigroup of $S$ generated by the idempotents of $S$. Then $C(S)$ is a completely regular subsemigroup of $S$ called the core of $S$. The operator $C$ is defined in $\mathscr{L}(\mathscr{C} \mathscr{R})$ by:

$$
\mathscr{V} C=\{S \in \mathscr{C} \mathscr{R} \mid C(S) \in \mathscr{V}\}
$$

It is routine to verify that $\mathscr{V} C$ is closed under products, homomorphic images and (completely regular) subsemigroups and is, therefore, a variety. Clearly $(\mathscr{V} C) C=\mathscr{V} C$ so that $C^{2}=C$. The operator $C$ appeared in a special case in ([13], Proposition 3.5).

The operator $L$ is defined on $\mathscr{L}(\mathscr{C} \mathscr{R})$ as follows:

$$
\mathscr{V} L=\{S \in \mathscr{C} \mathscr{R} \mid e S e \in \mathscr{V} \text { for all } e \in E(S)\}
$$


This operator was introduced in [3]; see ([3], Proposition 4.1) where it is shown that $\mathscr{V} L \in \mathscr{L}(\mathscr{C} \mathscr{R})$ and that $(\mathscr{V} L) L=\mathscr{V} L$ or $L^{2}=L$. Its restriction to $\mathscr{L}(\mathscr{C} \mathscr{S})$ was considered in $([12], \S 4)$.

The calculation of the semigroup generated by $C$ and $L$ is quite simple as we shall now see.

LEMMA 3.1. $L C L=C L$.

Proof. For $\mathscr{V} \in \mathscr{L}(\mathscr{C} \mathscr{R})$ and $S \in \mathscr{V} L C L$, we successively obtain

$$
\begin{aligned}
e S e & \in \mathscr{V} L C & & \text { for all } e \in E(S), \\
C(e S e) & \in \mathscr{V} L & & \text { for all } e \in E(S), \\
f C(e S e) f & \in \mathscr{V} & & \text { for all } e \in E(S) \text { and } f \in E(e S e), \\
e C(e S e) e & \in \mathscr{V} & & \text { for all } e \in E(S), \\
C(e S e) & \in \mathscr{V} & & \text { for all } e \in E(S), \\
e S e & \in \mathscr{V} C & & \text { for all } e \in E(S), \\
S & \in \mathscr{V} C L, & &
\end{aligned}
$$

so that $\mathscr{V} L C L \subseteq \mathscr{V} C L$; the opposite inclusion being trivial, we obtain the desired equality.

The next lemma is valid in any regular semigroup.

Lemma 3.2. If $e \in E(S)$ and $S$ is a regular semigroup, then $C(e S e)=$ $C(e C(S) e)$.

Proof. Let $x \in C(e S e)$. Then $x=e_{1} e_{2} \cdots e_{n}$ for some $e_{i} \in$ $E(e C(S) e)$ so that $x \in C(e C(S) e)$. This proves that $C(e S e) \subseteq$ $C(e C(S) e)$; the opposite inclusion is obvious.

LEMMA 3.3. $C L C=C L$.

Proof. For $\mathscr{V} \in \mathscr{L}(\mathscr{C} \mathscr{R})$ and $S \in \mathscr{V} C L C$, we successively obtain

$$
\begin{aligned}
C(S) & \in \mathscr{V} C L & & \\
e C(S) e & \in \mathscr{V} C & & \text { for all } e \in E(S), \\
C(e C(S) e) & \in \mathscr{V} & & \text { for all } e \in E(S), \\
C(e S e) & \in \mathscr{V} & & \text { for all } e \in E(S) \text { by Lemma 3.2, } \\
e S e & \in \mathscr{V} C & & \text { for all } e \in E(S), \\
S & \in \mathscr{V} C L, & &
\end{aligned}
$$

so that $\mathscr{V} C L C \subseteq \mathscr{V} C L$; the opposite inclusion being trivial, the desired equality follows. 
Applying $C, L, L C$ and $C L$ to the trivial variety $\mathscr{T}$, we see that they are all distinct. Then Lemmas 3.1 and 3.3 imply that $[C, L]=$ $\{C, L, L C, C L\}$ with the multiplication table

\begin{tabular}{c|c|c|c|c|} 
& $C$ & $L$ & $L C$ & $C L$ \\
\hline$C$ & $C$ & $C L$ & $C L$ & $C L$ \\
\hline$L$ & $L C$ & $L$ & $L C$ & $C L$ \\
\hline$L C$ & $L C$ & $C L$ & $C L$ & $C L$ \\
\hline$C L$ & $C L$ & $C L$ & $C L$ & $C L$ \\
\hline
\end{tabular}

As a particular case of ([3], Theorem 2), we have the following useful observation.

LEMmA 3.4. For $S \in \mathscr{C} \mathscr{R}$, we have $C(S)=\bigcup_{x \in S} C\left(D_{x}\right)$.

4. The kernel and trace operators. Of considerable importance in what follows is the anti-isomorphism from the lattice $\mathscr{L}(\mathscr{C} \mathscr{R})$ of subvarieties of $\mathscr{C} \mathscr{R}$ to the lattice $\mathscr{C}$ of fully invariant congruences on the free completely regular semigroup $F$ on a countably infinite set of generators. We denote this correspondence by

$$
\mathscr{V} \rightarrow \rho_{\mathscr{V}}, \quad \rho \rightarrow[\rho] \quad(\mathscr{V} \in \mathscr{L}(\mathscr{C} \mathscr{R}), \rho \in \mathscr{C}) .
$$

For any congruence $\rho$ on $S$ in $\mathscr{C} \mathscr{R}$,

$$
\begin{aligned}
\operatorname{ker} \rho & =\left\{x \in S \mid x \rho x^{0}\right\}, \\
\operatorname{tr} \rho & =\left.\rho\right|_{E(S)}, \\
\operatorname{tr} \rho & =\left.(\rho \vee \mathscr{L})^{0}\right|_{E(S)}, \\
\operatorname{rtr} \rho & =\left.(\rho \vee \mathscr{R})^{0}\right|_{E(S)},
\end{aligned}
$$

and are called, respectively, the kernel, trace, left trace and right trace of $\rho$. Each of these objects determines an equivalence relation on the lattice Con $S$ :

$$
\begin{aligned}
\lambda K \rho & \Leftrightarrow \operatorname{ker} \lambda=\operatorname{ker} \rho, \\
\lambda T \rho & \Leftrightarrow \operatorname{tr} \lambda=\operatorname{tr} \rho, \\
\lambda T_{l} \rho & \Leftrightarrow \operatorname{lt} \lambda=\operatorname{lt} \rho, \\
\lambda T_{r} \rho & \Leftrightarrow \operatorname{rtr} \lambda=\operatorname{rtr} \rho .
\end{aligned}
$$

The last two relations were introduced in $([9], \S 6)$.

Two fundamental observations on these relations are:

(i) ([1], Theorem 4.1). $K \cap T=\varepsilon$;

(ii) ([9], Theorem 6.12). $T_{l} \cap T_{r}=T$. 
In what follows we will only be interested in the restrictions of these relations to $\mathscr{C}$. We will denote these restrictions by the same symbols $K, T, T_{1}$ and $T_{r}$.

LEMmA 4.1. The relations $K, T, T_{l}$ and $T_{r}$ are complete congruences on $\mathscr{C}$.

Proof. See ([8], Theorem 11), ([9], Theorem 6.6).

If $\rho \in \mathscr{C}$, then it follows from Lemma 4.1 that each of the classes $\rho K, \rho T, \rho T_{r}$ and $\rho T_{l}$ has a minimum member which we shall denote by $\rho_{K}, \rho_{T}, \rho_{T_{i}}$ and $\rho_{T_{r}}$, respectively. We can repeat this process to obtain a network of congruences $\rho_{K T}, \rho_{K T K}, \rho_{K T_{l}}, \rho_{T_{l} T_{r}}$ etc.

In combination with the duality between varieties and fully invariant congruences, this enables us to introduce four operators on $\mathscr{L}(\mathscr{C} \mathscr{R})$ defined as follows: for any $\rho \in \mathscr{C}$,

$$
[\rho] K=\left[\rho_{K}\right], \quad[\rho] T=\left[\rho_{T}\right], \quad[\rho] T_{l}=\left[\rho_{T_{l}}\right], \quad[\rho] T_{r}=\left[\rho_{T_{r}}\right] .
$$

Clearly $K, T, T_{l}$ and $T_{r}$ are closure operators. In particular,

$$
K^{2}=K, \quad T^{2}=T, \quad T_{l}^{2}=T_{l}, \quad T_{r}^{2}=T_{r} .
$$

In order to work with these operators effectively, it is necessary to have alternative descriptions of $\mathscr{V} K, \mathscr{V} T$ etc. Toward this end, the following concept will prove useful.

Let $\mathscr{U}, \mathscr{V} \in \mathscr{L}(\mathscr{C} \mathscr{R})$. The Mal'cev product $\mathscr{U} \circ \mathscr{V}$ of $\mathscr{U}$ and $\mathscr{V}$ is defined by

$$
\begin{array}{r}
\mathscr{U} \circ \mathscr{V}=\left\{S \in \mathscr{C} \mathscr{R} \mid \text { there exists } \rho \in \text { Con } S \text { such that (i) if }(x \rho)^{2}=x \rho,\right. \\
\text { then } x \rho \in \mathscr{U} \text { and (ii) } S / \rho \in \mathscr{V}\} .
\end{array}
$$

In general $\mathscr{U} \circ \mathscr{V}$ need not be a variety ([7], Theorem 3.1).

LEMMA 4.2. ([7], Theorems 4.1 and 5.1).

(i) If $\mathscr{U}, \mathscr{V} \in \mathscr{L}(\mathscr{C} \mathscr{S})$, then $\mathscr{U} \circ \mathscr{V} \in \mathscr{L}(\mathscr{C} \mathscr{S})$.

(ii) If $\mathscr{U} \in \mathscr{L}(\mathscr{R} \mathscr{B} \vee \mathscr{G})$ and $\mathscr{S} \subseteq \mathscr{V}$, then $\mathscr{U} \circ \mathscr{V} \in \mathscr{L}(\mathscr{C} \mathscr{R})$.

LEMMA 4.3. Let $\mathscr{V} \in \mathscr{L}(\mathscr{C} \mathscr{R})$.

(i) $\mathscr{V} K$

$$
\begin{aligned}
& =\mathscr{R} \mathscr{B} \circ(\mathscr{V} \vee \mathscr{S})=\mathscr{B} \circ(\mathscr{V} \vee \mathscr{S}) \\
& =\{S \in \mathscr{C} \mathscr{R} \mid S / \tau \in \mathscr{V} \vee \mathscr{S}\} \\
& =\{S \in \mathscr{C} \mathscr{R} \mid S /(\tau \cap \mathscr{D}) \in \mathscr{V} \vee \mathscr{S}\} .
\end{aligned}
$$


(ii) $\mathscr{V} T=\mathscr{G} \circ \mathscr{V}=\{S \in \mathscr{C} \mathscr{R} \mid S / \mu \in \mathscr{V}\}$.

(iii) $\mathscr{V} T_{l}=\mathscr{L} \mathscr{G} \circ \mathscr{V}=\left\{S \in \mathscr{C} \mathscr{R} \mid S / \mathscr{L}^{0} \in \mathscr{V}\right\}$.

(iv) $\mathscr{V} T_{r}=\mathscr{R} \mathscr{G} \circ \mathscr{V}=\left\{S \in \mathscr{C} \mathscr{R} \mid S / \mathscr{R}^{0} \in \mathscr{V}\right\}$.

Proof. See ([7], Proposition 6.1 and Lemma 3.2) and ([9], Theorem $6.3)$.

THEOREM 4.4. In $F$, we have

$$
\tau=\mu=\mathscr{L}^{0}=\mathscr{R}^{0}=\varepsilon .
$$

Proof. It was established in ([10]), Lemma 5.11) that $\tau=\mu=\varepsilon$. Now suppose that $a, b \in F$ and $a \mathscr{L}^{0} b$. Let $T=\left\{x_{s} \mid s \in F^{1}\right\}$ and define a multiplication on $S=F \cup T$ by

$$
u v= \begin{cases}u v & \text { if } u, v \in F \\ x_{s} & \text { if } v=x_{s} \in T \\ x_{s v} & \text { if } u=x_{s} \in T, v \in F\end{cases}
$$

Then $S \in \mathscr{C} \mathscr{R}$. Since $S$ is countably infinite, it follows that there exists an epimorphism $\theta: F \rightarrow S$. Moreover, $\theta$ can be chosen to map the variables in $a$ and $b$ identically so that, in particular, $a \theta=a, b \theta=b$. Since $S$ is a homomorphic image of $F$, we have $a \mathscr{L}^{0} b$ in $S$. Hence

$$
x_{a}=x_{1 a}=x_{1} a \mathscr{L} x_{1} b=x_{1 b}=x_{b} .
$$

But $T$ is a right zero semigroup. Therefore $x_{a}=x_{b}$ and $a=b$. Thus $\mathscr{L}^{0}=\varepsilon$ on $F$ and, by duality, $\mathscr{R}^{0}=\varepsilon$.

CoRollary 4.5. If $\mathscr{V}$ is a proper subvariety of $\mathscr{C} \mathscr{R}$, then so are $\mathscr{V} K, \mathscr{V} T, \mathscr{V} T_{l}$ and $\mathscr{V} T_{r}$.

Proof. For example, consider $\mathscr{V} T_{r}$. If $\mathscr{V} T_{r}=\mathscr{C} \mathscr{R}$, then $F \in \mathscr{V} T_{r}$ and so, by Lemma 4.3 (iv), $F / \mathscr{R}^{0} \in \mathscr{V}$. By Theorem $4.4, \mathscr{R}^{0}$ is the identity congruence. Thus $F \in \mathscr{V}$ and $\mathscr{V}=\mathscr{C} \mathscr{R}$.

The next result explains the local and global behaviour of these operators.

THEOREM 4.6. Let $\mathscr{V} \in \mathscr{L}(\mathscr{C} \mathscr{R}), \mathscr{V} \neq \mathscr{C} \mathscr{R}$.

(i) $\mathscr{V} \subset \mathscr{V} K T \subset \mathscr{V}(K T)^{2} \subset \cdots \subset \mathscr{C} \mathscr{R}$ and $\vee \mathscr{V}(K T)^{n}=\mathscr{C} \mathscr{R}$.

(ii) $\mathscr{V} \subset \mathscr{V} K T_{l} \subset \mathscr{V}\left(K T_{l}\right)^{2} \subset \cdots \subset \mathscr{C} \mathscr{R}$ and $\vee \mathscr{V}\left(K T_{l}\right)^{n}=\mathscr{C} \mathscr{R}$.

(iii) If $\mathscr{S} \subseteq \mathscr{V}$, then $\mathscr{V} \subset \mathscr{V} T_{l} T_{r} \subset \mathscr{V}\left(T_{l} T_{r}\right)^{2} \subset \cdots \subset \mathscr{C} \mathscr{R}$ and $\mathrm{V} \mathscr{V}\left(T_{l} T_{r}\right)^{n}=\mathscr{C} \mathscr{R}$

Proof. It follows immediately from Corollary 4.5 that $\mathscr{V}(K T)^{n}$, $\mathscr{V}\left(K T_{l}\right)^{n}$ and $\mathscr{V}\left(T_{l} T_{r}\right)^{n}$ are proper subvarieties of $\mathscr{C} \mathscr{R}$ for all positive 
integers $n$. The remainder of part (i) can be found in ([10]), Corollary 5.9). From Lemma 4.3(ii) and (iii), it is clear that $\mathscr{V}(K T)^{n} \subseteq \mathscr{V}\left(K T_{l}\right)^{n}$ for all positive integers $n$, and so it follows from (i) that $\mathscr{C} \mathscr{R}=\mathrm{V} \mathscr{V}\left(K T_{l}\right)^{n}$. Now, if $\mathscr{V}\left(K T_{l}\right)^{m}=\mathscr{V}\left(K T_{l}\right)^{m+1}$ for some $m$, then clearly $\mathscr{V}\left(K T_{l}\right)^{m}=$ $\mathscr{V}\left(K T_{l}\right)^{k}$ for all $k \geq m$. But this would imply that $\mathscr{C} \mathscr{R}=\mathrm{V} \mathscr{V}\left(K T_{l}\right)^{n}=$ $\mathscr{V}\left(K T_{l}\right)^{m}$, which would contradict the fact that $\mathscr{V}\left(K T_{l}\right)^{n}$ is a proper subvariety of $\mathscr{C} \mathscr{R}$ for all $n$. Thus (ii) also holds. Now consider (iii).

Let $\mathscr{S} \subseteq \mathscr{V}$. In order to establish the last assertion, it suffices to show that $F_{k} \in \mathscr{S}\left(T_{l} T_{r}\right)^{k}$ for all positive integers $k$, where $F_{k}$ is the free completely regular semigroup on $k$ generators.

It is clear from Lemma 4.3(iii) that

$$
\mathscr{G} \subseteq \mathscr{L} \mathscr{G} \circ \mathscr{S}=\mathscr{S} T_{l} \subseteq \mathscr{S} T_{l} T_{r}
$$

so that $F_{k} \in \mathscr{S}\left(T_{l} T_{r}\right)^{k}$ holds for $k=1$. Now suppose that $F_{k} \in \mathscr{S}\left(T_{l} T_{r}\right)^{k}$ and consider $F_{k+1}$.

Let $K$ be the minimum component of $F_{k+1}$ and define $\rho$ on $F_{k+1}$ by

$$
a \rho b \Leftrightarrow\left\{\begin{array}{l}
a, b \in K \text { and } a \mathscr{R} b \\
a=b \text { otherwise. }
\end{array}\right.
$$

Then $\rho$ is a congruence on $F_{k+1}$ and $\rho \subseteq \mathscr{R}$. Now let $\sigma$ be the Rees congruence on $F_{k+1} / \rho$ determined by the ideal $K / \rho$ of $F_{k+1} / \rho$. Since $K / \rho$ is a single $\mathscr{L}$-class of $F_{k+1} / \rho$, we have $\sigma \subseteq \mathscr{L}$. But $\left(F_{k+1} / \rho\right) / \sigma \cong$ $F_{k+1} / K$, the Rees quotient for $F_{k+1}$ determined by the ideal $K$.

For each component $D$ of $F_{k+1} / K$ covering the zero component, let $\bar{D}$ be the union of all components above it. On $F_{k+1} / K$ define a relation $\rho_{D}$ by

$$
a \rho_{D} b \Leftrightarrow a=b \quad \text { or } \quad a, b \notin \bar{D} .
$$

Then $\rho_{D}$ is a congruence on $F_{k+1} / K$ and $\left(F_{k+1} / K\right) / \rho_{D} \cong F_{k}^{0}$.

In addition, the intersection of all congruences $\rho_{D}$ is the equality relation. We conclude that $F_{k+1} / K$ is a subdirect product of copies of $F_{k}^{0}$. Since $F_{k}$, and therefore $F_{k}^{0}$, lies in $\mathscr{S}\left(T_{l} T_{r}\right)^{k}$, it follows that $F_{k+1} / K \in$ $\mathscr{S}\left(T_{l} T_{r}\right)^{k}$. Thus $F_{k+1} / \rho \in \mathscr{S}\left(T_{l} T_{r}\right)^{k} T_{l}$ and $F_{k+1} \in \mathscr{S}\left(T_{l} T_{r}\right)^{k} T_{l} T_{r}=$ $\mathscr{S}\left(T_{l} T_{r}\right)^{k+1}$, as required. Hence $\vee \mathscr{V}\left(T_{l} T_{r}\right)^{n}=\mathscr{C} \mathscr{R}$. It now follows, as in part (ii), that $\mathscr{V}\left(T_{l} T_{r}\right)^{n} \subset \mathscr{V}\left(T_{l} T_{r}\right)^{n+1}$ for all positive integers $n$.

In Theorem 4.6 , it is shown that $\mathscr{C} \mathscr{R}$ is the join of an infinite family of proper subvarieties. In contrast, we have the following result.

THEOREM 4.7. The variety $\mathscr{C} \mathscr{R}$ is finitely join irreducible.

Proof. Let $\mathscr{U}, \mathscr{V} \in \mathscr{L}(\mathscr{C} \mathscr{R}), \mathscr{U} \neq \mathscr{C} \mathscr{R}, \mathscr{V} \neq \mathscr{C} \mathscr{R}$. Then there must exist a positive integer $n$ such that $F_{n} \notin \mathscr{U} \cup \mathscr{V}$. Suppose that $F_{n} \in \mathscr{U} \vee \mathscr{V}$. 
Then there exist $A \in \mathscr{U}, B \in \mathscr{V}$, a subdirect product $R$ of $A$ and $B$ and an epimorphism $\theta: R \rightarrow F_{n}$. Since $F_{n}$ is the free completely regular semigroup on $n$ generators, there exists a homomorphism $\varphi: F_{n} \rightarrow R$ such that $\iota=\varphi \theta$, where $\iota$ is the identity mapping on $F_{n}$ :

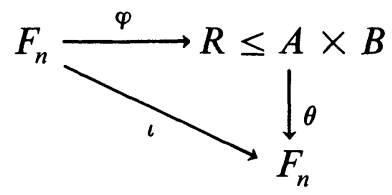

It follows that $\varphi$ is a monomorphism and we may consider $F_{n}$ as a subsemigroup of $A \times B$. Let $\pi_{A}$ and $\pi_{B}$ denote the projections of $A \times B$ onto $A$ and $B$, respectively, and let $\rho=\pi_{A} \circ \pi_{A}^{-1}, \sigma=\pi_{B} \circ \pi_{B}^{-1}$. Then $\rho \cap \sigma=\varepsilon$. Since $F_{n} \notin \mathscr{U} \cup \mathscr{V}, \rho$ and $\sigma$ are both non-trivial congruences.

Let $K$ be the minimum component of $F_{n}$ and $\kappa$ be the Rees congruence on $F_{n}$ determined by the ideal $K$. By the remark prior to Theorem 5.12 in [9], there are no idempotent pure congruences in $F_{n}$.

Hence, there exists $x \in F_{n} \backslash E\left(F_{n}\right)$ such that $x \rho x^{0}$. We prove next that there exists $k \in K$ such that $x k \neq x^{0} k$. If $x \in K$, take $k=x^{0}$. It remains to consider the case $x \notin K$. To this end, it suffices to construct a completely regular semigroup $S$ with an epimorphism $\theta: F_{n} \rightarrow S$ such that $(x k) \theta \neq\left(x^{0} k\right) \theta$. For this, let $S=F_{n-1} \cup\left\{y_{r} \mid r \in F_{n}^{1}\right\}$ with the multiplication

$$
a b= \begin{cases}a b & \text { if } a, b \in F_{n-1} \\ y_{r} & \text { if } a=y_{r} \\ y_{a r} & \text { if } b=y_{r} .\end{cases}
$$

Then $S \in \mathscr{C} \mathscr{R}$ and

$$
x y_{1}=y_{x 1}=y_{x} \neq y_{x^{0}}=y_{x^{0} 1}=x^{0} y_{1} .
$$

Let $x_{1}, x_{2}, \ldots, x_{n}$ be the free generators of $F_{n}$. Define a mapping $\theta$ by

$$
\theta: x_{i} \rightarrow x_{i} \text { for } i=1,2, \ldots, n-1, \quad x_{n} \rightarrow y_{1},
$$

and extend it to a homomorphism of $F_{n}$ into $S$. Letting

$$
k=x_{n}\left(x_{1} \cdots x_{n-1}\right)^{0},
$$

we obtain $k \in K$ and $k \theta=y_{1}\left(x_{1} \cdots x_{n-1}\right)^{0}=y_{1}$. Consequently, (2) gives $(x k) \theta \neq\left(x^{0} k\right) \theta$ and thus $x k \neq x^{0} k$. Since $x k \rho x^{0} k$, it follows that $\rho_{1}=\rho \cap \kappa$ is not the equality relation. Again by [9], $\rho_{1}$ is not idempotent pure. Therefore, there must be an element $a \in K \backslash E(K)$ with $a \rho_{1} a^{0}$.

Let $\sigma_{1}$ be similarly derived from $\sigma$. Then there is an element $b \in K \backslash E(K)$ with $b \sigma_{1} b^{0}$. Since $K$ is completely simple, we may assume that $a \mathscr{H} b$. The restrictions of $\rho_{1}$ and $\sigma_{1}$ to $H_{a}$, say $\rho_{2}$ and $\sigma_{2}$, are then 
both non-trivial. But $H_{x}$ is a free group ([2], Theorem 5.7) and so $\rho_{2} \cap \sigma_{2}$ is also non-trivial. Hence $\rho_{1} \cap \sigma_{1}$ is non-trivial and so also $\rho \cap \sigma$, which is a contradiction.

5. $C$ commutes with $K, T, T_{l}$ and $T_{r}$. In order to prove the statement in the title, we first consider how idempotent pure congruences restrict to the core.

Lemma 5.1. Let $S \in \mathscr{C} \mathscr{R}$ and $C=C(S)$. Then

$$
\left.\left(\tau_{S} \cap \mathscr{D}\right)\right|_{C}=\tau_{C} \cap \mathscr{D} \text {. }
$$

Proof. Let $\tau=\left.\left(\tau_{S} \cap \mathscr{D}\right)\right|_{C}$ and $\sigma=\tau_{C} \cap \mathscr{D}$. Clearly $\tau \subseteq \sigma$. Since $\sigma$ and $\tau$ are idempotent pure, it suffices to show that they have the same trace. So let $e, f \in E(S)=E$ and $e \sigma f$. Let $x, y \in S^{1}$ be such that $x e y \in E$. Then

$$
(e y x)^{2}=e y(x e y) x=e y(x e y)^{2} x=(e y x)^{3}
$$

so that, since eyx lies in a subgroup, eyx $\in E$. Thus $e(e y x) \in E$ and so, since $e \sigma f$ and eyx $\in C$, we must have $f($ eyx $) \in E$. Hence

$$
(y x f e)^{3}=y x(f e y x)(f e y x) f e=y x(f e y x) f e=(y x f e)^{2}
$$

and $y x f e \in E$. Thus $(y x f e) e \in E$ and, again since $e \sigma f$ and $y x f e \in C$, we obtain $y x f e f \in E$. But $e \mathscr{D} f$ so that $f \mathscr{H} f e f$. Since $e \tau_{C} f$ and $\tau_{C}$ restricted to any $\mathscr{H}$-class is trivial we get $f e f=f$. Therefore $y x f \in E$ and

$$
(x f y)^{3}=x f(y x f)(y x f) y=x f(y x f) y=(x f y)^{2} .
$$

Thus $x f y \in E$. By symmetry, we have that, for all $x, y \in S^{1}$,

$$
x e y \in E \Leftrightarrow x f y \in E
$$

and therefore $e \tau f$. Hence $\sigma=\tau$, as required.

LeMma 5.2. Let $\mathscr{V} \in \mathscr{L}(\mathscr{C} \mathscr{R})$. Then $(\mathscr{V} \vee \mathscr{S}) C=\mathscr{V} C \vee \mathscr{S}$.

Proof. The claim is trivial if $\mathscr{S} \subseteq \mathscr{V}$. So suppose that $\mathscr{S} \nsubseteq \mathscr{V}$ so that $\mathscr{V} \subseteq \mathscr{C} \mathscr{S}$.

We clearly have $\mathscr{V} C \vee \mathscr{S} \subseteq(\mathscr{V} \vee \mathscr{S}) C$. So now let $S \in(\mathscr{V} \vee \mathscr{S}) C$. Then $C(S) \in \mathscr{V} \vee \mathscr{S}$ and thus $C(S)$ is a normal band of groups. By ([11], IV.4.3), normal bands of groups can be characterized by the behaviour of their idempotents relative to their components. Since this pertains only to idempotents, it follows that $S$ itself is a normal band of groups. If $D$ is a 
component of $S$, then by Lemma 3.4 and ([6], Theorem 3.3), we obtain $C(D)=C(S) \cap D \in(\mathscr{V} \vee \mathscr{S}) \cap \mathscr{C} \mathscr{S}=(\mathscr{V} \cap \mathscr{C} \mathscr{S}) \vee(\mathscr{S} \cap \mathscr{C} \mathscr{S})=\mathscr{V}$.

Therefore $D \in \mathscr{V} C$ for each component $D$ of $S$ which together with $S$ being a normal band of groups and the proof of ([11], IV.4.3), yields that $S$ is a subdirect product of its components with a zero possibly adjoined. Since each of these, by the above, is contained in $\mathscr{V} C \vee \mathscr{S}$, we conclude that $S \in \mathscr{V} C \vee \mathscr{S}$.

We can now show that $C$ commutes with $K$.

LemMa 5.3. $K C=C K$.

Proof. First let $S \in \mathscr{C} \mathscr{R}$ and define a mapping $\chi$ by

$$
\chi: c\left(\tau_{S} \cap \mathscr{D}\right) \rightarrow c\left(\tau_{S} \cap \mathscr{D}\right) \cap C(S) \quad(c \in C(S)) .
$$

Lemma 5.1 asserts that $\left.\left(\tau_{S} \cap \mathscr{D}\right)\right|_{C(S)}=\tau_{C(S)} \cap \mathscr{D}$ which then implies that $\chi$ is a bijection of $C\left(S /\left(\tau_{S} \cap \mathscr{D}\right)\right)$ onto $C(S) /\left(\tau_{C(S)} \cap \mathscr{D}\right)$ since every homomorphism maps the core onto the core. It now follows by Lemma 5.1 that $\chi$ is also a homomorphism. Therefore

$$
C\left(S /\left(\tau_{S} \cap \mathscr{D}\right)\right) \cong C(S) /\left(\tau_{C(S)} \cap \mathscr{D}\right) .
$$

For $\mathscr{V} \in \mathscr{L}(\mathscr{C} \mathscr{R})$, we obtain

$$
\begin{array}{rlrl}
S \in \mathscr{V} K C & \Leftrightarrow C(S) \in \mathscr{V} K & \\
& \Leftrightarrow C(S) /\left(\tau_{C(S)} \cap \mathscr{D}\right) \in \mathscr{V} \vee \mathscr{S} & & \text { by Lemma } 4.3 \\
& \Leftrightarrow C\left(S /\left(\tau_{S} \cap \mathscr{D}\right)\right) \in \mathscr{V} \vee \mathscr{S} & & \text { by }(3) \\
& \Leftrightarrow S /\left(\tau_{S} \cap \mathscr{D}\right) \in(\mathscr{V} \vee \mathscr{S}) C & \\
& \Leftrightarrow S /\left(\tau_{S} \cap \mathscr{D}\right) \in \mathscr{V} C \vee \mathscr{S} & & \text { by Lemma } 5.2 \\
& \Leftrightarrow S \in \mathscr{V} C K & & \text { by Lemma 4.3. }
\end{array}
$$

Thus $\mathscr{V} K C=\mathscr{V} C K$, as required.

LeMmA 5.4. Let $S \in \mathscr{C} \mathscr{R}$ and $C=C(S)$. Then

$$
\left.\mathscr{L}^{0}\right|_{C}=\left(\mathscr{L}_{C}\right)^{0},\left.\quad \mathscr{R}^{0}\right|_{C}=\left(\mathscr{R}_{C}\right)^{0},\left.\quad \mu\right|_{C}=\mu_{C} .
$$

Proof. Consider $\mathscr{L}^{0}$. Clearly $\left.\mathscr{L}^{0}\right|_{C} \subseteq\left(\mathscr{L}_{C}\right)^{0}$. So let $a, b \in C, a \mathscr{L}_{C}^{0} b$ and $x, y \in S^{1}$. Then $x^{0} a y^{0} \mathscr{L}_{C} x^{0} b y^{0}$ since $a \mathscr{L}_{C}^{0} b$, and $x^{0} a y \mathscr{L} x^{0}$ by since $\mathscr{L}$ is a right congruence. But clearly

$$
x a y \mathscr{L} x^{0} a y, \quad x b y \mathscr{L} x^{0} b y
$$

so that $x a y \mathscr{L} x$ by. Thus $a\left(\left.\mathscr{L}^{0}\right|_{C}\right) b$ and $\left.\mathscr{L}^{0}\right|_{C}=\left(\mathscr{L}_{C}\right)^{0}$. Similarly for $\mathscr{R}$ and $\mu=\mathscr{H}^{0}=\mathscr{R}^{0} \cap \mathscr{L}^{0}$. 
LEMMA 5.5. $T C=C T, T_{l} C=C T_{l}, T_{r} C=C T_{r}$.

Proof. Consider $T_{l}$. From Lemma 5.4, it follows that

$$
C\left(S / \mathscr{L}^{0}\right) \cong C(S) / \mathscr{L}_{C(S)}^{0} .
$$

For any $\mathscr{V} \in \mathscr{L}(\mathscr{C} \mathscr{R})$, we then obtain

$$
\begin{aligned}
S \in \mathscr{V} T_{l} C & \Leftrightarrow C(S) \in \mathscr{V} T_{l} \Leftrightarrow C(S) / \mathscr{L}_{C(S)}^{0} \in \mathscr{V} \\
& \Leftrightarrow C\left(S / \mathscr{L}^{0}\right) \in \mathscr{V} \Leftrightarrow S / \mathscr{L}^{0} \in \mathscr{V} C \Leftrightarrow S \in \mathscr{V} C T_{l} .
\end{aligned}
$$

Similarly for $T_{r}$ and $T$.

6. $L$ commutes with $K, T, T_{l}$ and $T_{r}$. In order to prove the statement in the title, we require information on the restriction of congruences to certain submonoids.

LemMA 6.1. For any $S \in \mathscr{C} \mathscr{R},\left.\tau_{S}\right|_{e S e}=\tau_{e S e}$ for all $e \in E(S)$.

Proof. Let $e \in E(S), \sigma=\left.\tau_{S}\right|_{e S e}$ and $\tau=\tau_{e S e}$. Clearly $\sigma \subseteq \tau$. For the opposite inclusion, it suffices to show that $\operatorname{tr} \tau \subseteq \operatorname{tr} \sigma$. Hence let $f, g \in E$ $=E(e S e)$ with $f \tau g$. Let $x, y \in S^{1}$ and assume that $x f y \in E(S)$. Then

$$
\begin{aligned}
{[f(\text { eyxe })]^{3} } & =f e y(x e f e y)^{2} x e=f e y(x f y)^{2} x e \\
& =f e y(x f y) x e=(f e y x e)^{2}
\end{aligned}
$$

which implies that $f($ eyxe $) \in E$, since $f($ eyxe $)$ is contained in a subgroup of $e S e$. The hypothesis implies that $g($ eyxe $) \in E(S)$ whence

$$
(x g y)^{3}=x(\text { geyxe })^{2} g y=x(\text { geyxe }) g y=(x g y)^{2}
$$

so that $x g y \in E(S)$. By symmetry, we deduce that fog. Consequently $\sigma=\tau$, as required.

LeMma 6.2. Let $\mathscr{V} \in \mathscr{L}(\mathscr{C} \mathscr{R})$. Then $(\mathscr{V} \vee \mathscr{S}) L=\mathscr{V} L \vee \mathscr{S}$.

Proof. The claim is trivial if $\mathscr{S} \subseteq \mathscr{V}$. So suppose that $\mathscr{V} \subseteq \mathscr{C} \mathscr{S}$. It is clear that $\mathscr{V} L \vee \mathscr{S} \subseteq(\mathscr{V} \vee \mathscr{S}) L$.

Let $S \in(\mathscr{V} \vee \mathscr{S}) L$. Then, for all $e \in E(S), e S e \in \mathscr{V} \vee \mathscr{S}$. It follows that $e S e$ is a normal band of groups. By ([11], IV.4.3), normal bands of groups can be characterized by the behaviour of their idempotents relative to the subsets $e S e$ as $e$ runs over all idempotents of $S$. It follows from this reference that since $e S e$ is a normal band of groups for all $e \in E(S)$, we have that $S$ itself is a normal band of groups. Moreover, for any $e \in E(S)$, using ([6], Theorem 3.3), we obtain

$$
e D_{e} e \in(\mathscr{V} \vee \mathscr{S}) \cap \mathscr{C} \mathscr{S}=(\mathscr{V} \cap \mathscr{C} \mathscr{S}) \vee(\mathscr{S} \cap \mathscr{C} \mathscr{S})=\mathscr{V},
$$


and thus $D_{e} \in \mathscr{V} L$. The proof of ([11], IV.4.3) implies that $S$ is a subdirect product of the semigroups $D_{e}$ with a zero possibly adjoined. Since each of these, by the above, is contained in $\mathscr{V} L \vee \mathscr{S}$, we conclude that $S \in \mathscr{V} L \vee \mathscr{S}$.

\section{LEMMA 6.3. $K L=L K$.}

Proof. First let $S \in \mathscr{C} \mathscr{R}$ and $e \in E(S)$. Define a mapping $\chi$ by

$$
\chi: a \tau \rightarrow a \tau \cap e S e \quad(a \in e S e) .
$$

Lemma 6.1 asserts that $\left.\tau\right|_{e S e}=\tau_{e S e}$ which implies that $\chi$ is a bijection of $(e \tau)(S / \tau)(e \tau)$ onto $(e S e) / \tau_{e S e}$. It now follows, by Lemma 6.1, that $\chi$ is also a homomorphism. Therefore

$$
(e \tau)(S / \tau)(e \tau) \cong(e S e) / \tau_{e S e} .
$$

For $\mathscr{V} \in \mathscr{L}(\mathscr{C} \mathscr{R})$, we obtain

$$
\begin{array}{rlr}
S \in \mathscr{V} K L & \Leftrightarrow e S e \in \mathscr{V} K \text { for all } e \in E(S) & \\
& \Leftrightarrow(e S e) / \tau_{e S e} \in \mathscr{V} \vee \mathscr{S} \text { for all } e \in E(S) \\
& \Leftrightarrow(e \tau)(S / \tau)(e \tau) \in \mathscr{V} \vee \mathscr{S} \text { for all } e \in E(S) \text { by (4) } \\
& \Leftrightarrow S / \tau \in(\mathscr{V} \vee \mathscr{S}) L & \\
& \Leftrightarrow S / \tau \in \mathscr{V} L \vee \mathscr{S} & \\
& \Leftrightarrow S \in \mathscr{V} L K & \text { by Lemma } 6.2
\end{array}
$$

Thus $\mathscr{V} K L=\mathscr{V} L K$ and the proof is complete.

We now consider the interaction of $L$ and $T, T_{l}$ and $T_{r}$.

Lemma 6.4. Let $S \in \mathscr{C} \mathscr{R}$ and $e \in E(S)$. Then

$$
\left.\mu\right|_{e S e}=\mu_{e S e},\left.\quad \mathscr{L}^{0}\right|_{e S e}=\left(\mathscr{L}_{e S e}\right)^{0},\left.\quad \mathscr{R}^{0}\right|_{e S e}=\left(\mathscr{R}_{e S e}\right)^{0} .
$$

Proof. Consider $\mathscr{L}^{0}$. Clearly $\left.\mathscr{L}^{0}\right|_{e S e} \subseteq\left(\mathscr{L}_{e S e}\right)^{0}$. So let $a, b \in e S e$, $a\left(\mathscr{L}_{e S e}\right)^{0} b$ and $x, y \in S$. First note that, for any $w \in S$,

$$
\begin{aligned}
(\text { exe })^{0}(\text { xe })^{0}(\text { exe }) w & =(\text { exe })^{-1}(\text { exe })(\text { xe })^{0} \text { exew } \\
& =(\text { exe })^{-1}(\text { exe })(\text { exe }) w=(\text { exe }) w
\end{aligned}
$$


so that $($ exe $) w \mathscr{L}(x e)^{0}($ exe $) w$. Also

$$
\begin{aligned}
& a\left(\mathscr{L}_{\text {eSe }}\right)^{0} b \Rightarrow(\text { exe }) a(\text { eye }) \mathscr{L}_{\text {eSe }}(\text { exe }) b(\text { eye }) \\
& \Rightarrow(x e)^{0}(\text { exe }) a(\text { eye }) \mathscr{L}(x e)^{0}(\text { exe }) b(\text { eye }) \text { by the above remarks } \\
& \text { since } \mathscr{L} \text { is a right congruence } \\
& \Rightarrow(x e)^{0} x e a e y(e y)^{0} \mathscr{L}(x e)^{0} x e b e y(e y)^{0} \\
& \Rightarrow \text { xeaey } \mathscr{L} \text { xebey } \\
& \Rightarrow \text { xay } \mathscr{L} \text { xby. }
\end{aligned}
$$$$
\Rightarrow(x e)^{0}(\text { exe }) a(\text { eye })(\text { ey })^{0} \mathscr{L}(x e)^{0}(\text { exe }) b(\text { eye })(\text { ey })^{0}
$$

Thus $a \mathscr{L}^{0} b$. The case of $\mathscr{R}$ is symmetric and the case of $\mu$ follows from these two cases.

LEMMA 6.5. $T L=L T, T_{l} L=L T_{l}, T_{r} L=L T_{r}$.

Proof. Consider $T_{l}$. From Lemma 6.4 it follows that

$$
\left(e \mathscr{L}^{0}\right)\left(S / \mathscr{L}^{0}\right)\left(e \mathscr{L}^{0}\right) \cong(e S e) / \mathscr{L}_{e S e}^{0} .
$$

Hence for any $\mathscr{V} \in \mathscr{L}(\mathscr{C} \mathscr{R})$, we obtain

$$
\begin{array}{rlrl}
S \in \mathscr{V} T_{l} L & \Leftrightarrow e S e \in \mathscr{V} T_{l} & & \text { for all } e \in E(S) \\
& \Leftrightarrow e S e / \mathscr{L}_{e S e}^{0} \in \mathscr{V} & & \text { for all } e \in E(S) \\
& \Leftrightarrow\left(e \mathscr{L}^{0}\right)\left(S / \mathscr{L}^{0}\right)\left(e \mathscr{L}^{0}\right) \in \mathscr{V} & & \text { for all } e \in E(S) \\
& \Leftrightarrow S / \mathscr{L}^{0} \in \mathscr{V} L \Leftrightarrow S \in \mathscr{V} L T_{l} . &
\end{array}
$$

Thus $T_{l} L=L T_{l}$. That $T_{r} L=L T_{r}$ and $T L=L T$ follows similarly.

7. Semigroups generated by any two of $K, T, T_{l}, T_{r}$. In this section we deal with some technical preparations for the determination of various semigroups of operators in terms of generators and relations. We shall require some additional notation.

For reasons that will soon be apparent, we will be interested mostly in the free semigroup on $\left\{c, l, k, t, t_{l}, t_{r}\right\}$. An element $w=a_{1} a_{2} \cdots a_{n} \in A^{+}$ will be called distinguished if $a_{i} \neq a_{i+1}$ for $i=1, \ldots, n-1$. The set of distinguished elements in $\{a, b, c, \ldots\}^{+}$will be denoted by $\Delta_{a b c} \ldots$.

If $v \in A^{+}$and we wish to emphasize the variables $x_{1}, \ldots, x_{n} \in A$ that appear in $w$, that we shall write $v\left(x_{1}, \ldots, x_{n}\right)$. If $S$ is a semigroup and 
$s_{1}, \ldots, s_{n} \in S$, then we shall denote by $v\left(s_{1}, \ldots, s_{n}\right)$ the element obtained from $v$ by substituting $s_{i}$ for $x_{i}, i=1, \ldots, n$.

In keeping with the notation $\rho_{K}$ and $\rho_{T}$ already introduced for the minimum congruence in the kernel and trace class of any congruence $\rho$ on a completely regular semigroup $S$, we will now denote by $\rho^{K}$ the maximum congruence in the kernel class of $\rho$; that is, $\rho^{K}$ denotes the largest congruence on $S$ with the same kernel as $\rho$. That $\rho^{K}$ exists follows from Lemma 4.1 and basic information about $\rho^{K}$ can be found in [9]. (Of course there is the dual concept of the maximum congruence $\rho^{T}$ in the trace class of $\rho$, but we shall not require that here.)

The variety $\mathscr{R} g \mathscr{B}$ of regular bands, that is, the variety of bands defined by the identity axya = axaya, has a role to play in the next two results.

Proposition 7.1 (Kaďourek [8], Proposition 8.2). For any $\rho \in \mathscr{C}$, with $\rho \subseteq \rho_{\mathscr{R} \mathscr{B}},\left(\rho_{T}\right)^{K}=\rho_{T}$.

LEMMA 7.2. Let $\mathscr{U}, \mathscr{V} \in \mathscr{L}(\mathscr{C} \mathscr{R}), \mathscr{R} g \mathscr{B} \subseteq \mathscr{V}$ and $\mathscr{V} \nsubseteq \mathscr{U}$. Then $\mathscr{V} T \nsubseteq \mathscr{U} K, \mathscr{V} T, \nsubseteq \mathscr{U} K$ and $\mathscr{V} T_{r} \nsubseteq \mathscr{U} K$.

Proof. Let $\mathscr{U}, \mathscr{V} \in \mathscr{L}(\mathscr{C} \mathscr{R})$ be such that $\mathscr{R} g \mathscr{B} \subseteq \mathscr{V}, \mathscr{V} \nsubseteq \mathscr{U}$ and suppose that $\mathscr{V} T \subseteq \mathscr{U} K$. Let $\rho=\rho_{\mathscr{U}}$ and $\sigma=\rho_{\mathscr{V}}$. Then $\sigma \subseteq \rho_{\mathscr{R}_{\mathscr{g}}}$ and $\rho_{K} \subseteq \sigma_{T}$. By $\left([10]\right.$, Theorem 4.5) the mapping $\rho \rightarrow \rho^{K}(\rho \in \mathscr{C})$ is order preserving. Hence

$$
\begin{aligned}
\rho_{K} \subseteq \sigma_{T} & \Rightarrow \rho^{K}=\left(\rho_{K}\right)^{K} \subseteq\left(\sigma_{T}\right)^{K} \\
& \Rightarrow \rho^{K} \subseteq\left(\sigma_{T}\right)^{K}=\sigma_{T} \quad \text { by Proposition 7.1 } \\
& \Rightarrow \rho \subseteq \rho^{K} \subseteq \sigma_{T} \subseteq \sigma \\
& \Rightarrow \mathscr{V} \subseteq \mathscr{U}
\end{aligned}
$$

a contradiction. This establishes the first claim. The claims that $\mathscr{V} T_{l} \nsubseteq \mathscr{U K}$ and $\mathscr{V} T_{r} \nsubseteq \mathscr{U K}$ now follow from the fact that $\mathscr{V} T=\mathscr{V} T_{l} \cap \mathscr{V} T_{r}$.

LEMMA 7.3. Let $\mathscr{U}, \mathscr{V} \in \mathscr{L}(\mathscr{C} \mathscr{R})$ and $\mathscr{S} \subseteq \mathscr{V}, \mathscr{V} \nsubseteq \mathscr{U}$. Then $\mathscr{V} K \nsubseteq$ $\mathscr{U} T, \mathscr{V} K \nsubseteq \mathscr{U} T_{l}$ and $\mathscr{V} K \nsubseteq \mathscr{U} T_{r}$.

Proof. Let $S \in \mathscr{V} \backslash \mathscr{U}$. Then $S^{0} \in \mathscr{V} \backslash \mathscr{U}$ since $\mathscr{S} \subseteq \mathscr{V}$. Let $X=$ $\left\{x_{s} \mid s \in S^{1}\right\}$ and define a multiplication $*$ on $R=S \cup X$ by

$$
a * b= \begin{cases}a b & \text { if } a, b \in S, \\ x_{s} & \text { if } b=x_{s} \in X, \\ x_{s b} & \text { if } a=x_{s} \in X\end{cases}
$$


Then $R$ is an ideal extension of the right zero semigroup $X$ by $S^{0}$. Therefore $R \in \mathscr{V} K \backslash \mathscr{U}$.

However, suppose that $a, b \in S$ and $a \mathscr{L}^{0} b$. Then $x_{a}=x_{1} a \mathscr{L} x_{1} b=$ $x_{b}$. But $X$ is a right zero subsemigroup of $R$. Therefore $x_{a}=x_{b}$ and $a=b$. Similarly $\mathscr{L}^{0}$ must be the equality on $X$ and therefore on $R$. Thus $R / \mathscr{L}^{0} \cong R \notin \mathscr{U}$ so that $R \notin \mathscr{U} T_{l}$. Hence $\mathscr{V} K \notin \mathscr{U} T_{l}$. Since $\mathscr{U} T \subseteq \mathscr{U} T_{l}$, it follows that $\mathscr{V} K \nsubseteq \mathscr{U} T$ and, by duality, $\mathscr{V} K \nsubseteq \mathscr{U} T_{r}$.

LEMMA 7.4. Let $\mathscr{U} \in \mathscr{L}(\mathscr{C} \mathscr{R}), \mathscr{U} \neq \mathscr{C} \mathscr{R}, v, w \in \Delta_{k t}, V=v(K, T), W$ $=w(K, T)$ and $\mathscr{U} V=\mathscr{U} W$. Then either (i) $v=w$ or (ii) $v$ and $w$ end in the same letter and differ in length by at most one.

Proof. Let $\mathscr{W}=\mathscr{U} V=\mathscr{U} W$. By Corollary 4.5, $\mathscr{W} \neq \mathscr{C} \mathscr{R}$. Since $v, w \in$ $\Delta_{k t}, v$ and $w$ are both of length at least one. First suppose that $v$ and $w$ end in different letters. Without loss of generality, we can assume that $v=v_{1} t$ and $w=w_{1} k$ for some $v_{1}, w_{1} \in \Delta_{k t} \cup\{\varnothing\}$. Letting $V_{1}=v_{1}(K, T)$ and $W_{1}=w_{1}(K, T)$, we have

$$
\begin{aligned}
& \mathscr{W} T=\mathscr{U} V T=\mathscr{U} V_{1} T T=\mathscr{U} V_{1} T=\mathscr{U} V=\mathscr{W}, \\
& \mathscr{W} K=\mathscr{U} W K=\mathscr{U} W_{1} K K=\mathscr{U} W_{1} K=\mathscr{U} W=\mathscr{W},
\end{aligned}
$$

which, since $\mathscr{U} \neq \mathscr{C} \mathscr{R}$, contradicts Theorem 4.6(i). Therefore $v$ and $w$ must end in the same letter. If $|v|=|w|$, then since $v$ and $w$ are both distinguished elements (so that occurrences of $t$ and $k$ alternate) we must have $v=w$.

So suppose that $|v| \neq|w|$ and that $v$ and $w$ both end in $k$ (a similar argument will handle the case where both end in $t$ ). Without loss of generality, we may assume that $v$ is the shorter word. Suppose that $|v|+2 \leq|w|$. Since $v$ and $w$ both end in $k$ and are both distinguished elements of $\{k, t\}^{+}$, there must exist an element $u \in \Delta_{k t} \cup\{\varnothing\}$ such that $w=u v t k$. Thus

$$
\mathscr{W} \subseteq \mathscr{W} T K=\mathscr{U} V T K \subseteq \mathscr{U} u(K, T) V T K=\mathscr{U} W=\mathscr{W}
$$

so that $\mathscr{W}=\mathscr{W} T K$. Since $\mathscr{W} \neq \mathscr{C} \mathscr{R}$, this contradicts Theorem 4.6(i). Thus $v$ and $w$ can differ in length by at most one.

Lemma 7.5. Let $\mathscr{U} \in \mathscr{L}(\mathscr{C} \mathscr{R}), \mathscr{N} \mathscr{B} \subseteq \mathscr{U}, \mathscr{U} \neq \mathscr{C} \mathscr{R}, \mathscr{U} \neq \mathscr{U} K, \mathscr{U} \neq$ $\mathscr{U} T, v, w \in \Delta_{k t} \cup\{\varnothing\}$ and $\mathscr{U} v(K, T)=\mathscr{U} w(K, T)$. Then $v=w$.

Proof. By the hypothesis that $\mathscr{U} \neq \mathscr{U} T, \mathscr{U} \neq \mathscr{U} K$, we cannot have one of $v, w$ equal to $\varnothing$, but not the other. Therefore we may assume that $v, w \in \Delta_{k t}$. 
Suppose that $v \neq w$. By Lemma 7.4, we may assume that $|w|=|v|+1$ and that $v$ and $w$ end in the same letter. Without loss of generality, let $v$ and $w$ end in $k$. We may also assume that $v$ has been chosen to have the smallest length of any element in $\Delta_{k t}$ for which there exists $w \in \Delta_{k t}$ with $|w|=|v|+1$ and $\mathscr{U} v(K, T)=\mathscr{U} w(K, T)$.

Let $v=v_{1} k$ and $w=w_{1} k$, where $v_{1}, w_{1} \in \Delta_{k t}$. Let $V=v(K, T), V_{1}$ $=v_{1}(K, T), W=w(K, T)$ and $W_{1}=w_{1}(K, T)$. By the minimality of $|v|, \mathscr{U} V_{1} \neq \mathscr{U} W_{1}$. Since $v, v_{1}, w, w_{1}$ are distinguished, $v_{1}$ and $w_{1}$ must both end in $t$. Also, occurrences of $k$ and $t$ alternate in distinguished words and $\left|w_{1}\right|=\left|v_{1}\right|+1$. Hence, we must have $w_{1}=t v_{1}$ or $w_{1}=k v_{1}$. Thus either $\mathscr{U} V_{1} \subseteq \mathscr{U} T V_{1}=\mathscr{U} W_{1}$ or $\mathscr{U} V_{1} \subseteq \mathscr{U} K V_{1}=\mathscr{U} W_{1}$. In both cases we conclude that $\mathscr{U} V_{1} \subset \mathscr{U} W_{1}$ so that $\mathscr{U} W_{1} \nsubseteq \mathscr{U} V_{1}$.

Since $w_{1}$ ends in $t, w_{1}(K, T) T=w_{1}(K, T)$ and so $\mathscr{U} W_{1} T=\mathscr{U} W_{1}$. But $\mathscr{U} W_{1} \nsubseteq \mathscr{U} V_{1}$ implies $\mathscr{U} W_{1} T \nsubseteq \mathscr{U} V_{1} K$ by Lemma 7.2 , so that

$$
\mathscr{U} W_{1}=\mathscr{U} W_{1} T \nsubseteq \mathscr{U} V_{1} K=\mathscr{U} V=\mathscr{U} W=\mathscr{U} W_{1} K
$$

which is a clear contradiction.

LEMMA 7.6. Let $\mathscr{U} \in \mathscr{L}(\mathscr{C} \mathscr{R})$.

(i) $\mathscr{U} T, \cap \mathscr{B}=\mathscr{L} \mathscr{Z} \circ(\mathscr{U} \cap \mathscr{B})$.

(ii) $\mathscr{U} T_{r} \cap \mathscr{B}=\mathscr{R} \mathscr{Z} \circ(\mathscr{U} \cap \mathscr{B})$.

Proof. By duality it suffices to prove (i). By Lemma 4.3(iii), we have $\mathscr{L} \mathscr{Z} \circ(\mathscr{U} \cap \mathscr{B}) \subseteq \mathscr{L} \mathscr{G} \circ \mathscr{U}=\mathscr{U} T_{l}$, while clearly $\mathscr{L} \mathscr{Z} \circ(\mathscr{U} \cap \mathscr{B}) \subseteq \mathscr{B}$. Thus $\mathscr{L} \mathscr{Z} \circ(\mathscr{U} \cap \mathscr{B}) \subseteq \mathscr{U} T_{l} \cap \mathscr{B}$. So let $S \in \mathscr{U} T_{l} \cap \mathscr{B}$. Then $S / \mathscr{L}^{0} \in \mathscr{U}, S \in$ $\mathscr{B}$ and $e \mathscr{L}^{0} \in \mathscr{L} \mathscr{G}$ for all $e \in E(S)$. Hence $e \mathscr{L}^{0} \in \mathscr{L} \mathscr{G} \cap \mathscr{B}=\mathscr{L} \mathscr{Z}$ for all $e \in E(S)$, and $S / \mathscr{L}^{0} \in \mathscr{U} \cap \mathscr{B}$. Thus $S \in \mathscr{L} \mathscr{Z} \circ(\mathscr{U} \cap \mathscr{B})$, as required and the equality in (i) holds.

\section{LEMMA 7.7. $T T_{l}=T_{l} T=T_{l}, T T_{r}=T_{r} T=T_{r}$.}

Proof. It suffices to consider $T_{l}$. Let $\mathscr{U} \in \mathscr{L}(\mathscr{C} \mathscr{R})$. Clearly $\mathscr{U} T_{l} \subseteq$ $\mathscr{U} T T_{l}$. So let $S \in \mathscr{U} T T_{l}$. Then $R=S / \mathscr{L}^{0} \in \mathscr{U} T$ and $R / \mu_{R} \in \mathscr{U}$. But $\mathscr{L}_{R}^{0}$ is the equality congruence on $R$ and, therefore, so also is $\mu_{R}$. Hence

$$
S / \mathscr{L}_{S}^{0}=R \cong R / \mu_{R} \in \mathscr{U}
$$

and so $S \in \mathscr{U} T_{l}$. Thus $\mathscr{U} T T_{l}=\mathscr{U} T_{l}$ and therefore $T T_{l}=T$.

It is also clear that $\mathscr{U} T_{l} \subseteq \mathscr{U} T_{l} T$. So let $S \in \mathscr{U} T_{l} T$. Then $R=S / \mu_{S}$ $\in \mathscr{U} T_{1}$ and $R / \mathscr{L}_{R}^{0} \in \mathscr{U}$. Since $\mu_{S} \subseteq \mathscr{L}_{S}^{0}$, it is easily seen that $\mathscr{L}_{R}^{0}=$ $\mathscr{L}_{S}^{0} / \mu_{S}$. Thus $S / \mathscr{L}_{S}^{0} \cong R / \mathscr{L}_{R}^{0} \in \mathscr{U}$ and $S \in \mathscr{U} T_{l}$. Hence $\mathscr{U} T_{l} T=\mathscr{U} T_{l}$ so that $T_{l} T=T_{l}$, as required. 
We are finally ready for the main result of this section.

THEOREM 7.8. (i) $[K, T] \cong\left\langle k, t \mid k^{2}=k, t^{2}=t\right\rangle$.

(ii) $\left[K, T_{l}\right] \cong\left\langle k, t_{l} \mid k^{2}=k, t_{l}^{2}=t_{l}\right\rangle$.

(iii) $\left[K, T_{r}\right] \cong\left\langle k, t_{r} \mid k^{2}=k, t_{r}^{2}=t_{r}\right\rangle$.

(iv) $\left[T_{l}, T_{r}\right] \cong\left\langle t_{l}, t_{r} \mid t_{l}^{2}=t_{l}, t_{r}^{2}=t_{r}\right\rangle$.

(v) $\left[T, T_{l}\right] \cong\left\langle t, t_{l} \mid t^{2}=t, t_{l}^{2}=t_{l}, t t_{l}=t_{l} t=t_{l}\right\rangle \cong\left\langle t_{l}\right\rangle^{1}$

(vi) $\left[T, T_{r}\right] \cong\left\langle t, t_{r} \mid t^{2}=t, t_{r}^{2}=t_{r}, t t_{r}=t_{r} t=t_{r}\right\rangle \cong\left\langle t_{r}\right\rangle^{1}$.

Proof. Let $\varphi:\{k, t\}^{+} \rightarrow[K, T]$ be the epimorphism defined by $k \varphi=$ $K, t \varphi=T$. Let $\rho=\varphi \circ \varphi^{-1}$ and $\sigma$ be the congruence on $\{k, t\}^{+}$generated by the relation $\left\{\left(k^{2}, k\right),\left(t^{2}, t\right)\right\}$. Since $K^{2}=K$ and $T^{2}=T$, it follows that $\sigma \subseteq \rho$. To establish the reverse inclusion, let $p, q \in\{k, t\}^{+}$and $p \rho q$. Using the relation defining $\sigma$, there exist $v, w \in \Delta_{k t}$ such that $p \sigma v$ and $q \sigma w$. Since $\sigma \subseteq \rho$, we have $v \rho w$. Thus $v(K, T)=v \varphi=w \varphi=w(K, T)$, and in particular $\mathscr{N} \mathscr{B} v(K, T)=\mathscr{N} \mathscr{B} w(K, T)$. But $\mathscr{N} \mathscr{B} K=\mathscr{B} \neq \mathscr{N} \mathscr{B}$ and $\mathscr{N} \mathscr{B} T=\mathscr{N} \mathscr{B} \mathscr{G} \neq \mathscr{N} \mathscr{B}$. Hence, by Lemma 7.5, $v=w$. Thus $p \sigma v=$ $w \sigma q$, so that $p \sigma q$ and $\rho \subseteq \sigma$. Therefore $\rho=\sigma$ and (i) follows.

The proofs of parts (ii) and (iii) are similar to that of (i). Lemmas 7.4 and 7.5 were derived from Lemmas 7.2 and 7.3. These latter lemmas hold equally well for $T_{l}$ and $T_{r}$ as for $T$. Therefore, throughout Lemmas 7.4 and 7.5 we may replace $T$ consistently by $T_{l}$ or by $T_{r}$ to obtain analogous results for $K$ combined with $T_{l}$ and for $K$ combined with $T_{r}$. The final parts of the proofs of (ii) and (iii) are then similar to that of (i) above.

We now prove part (iv). Let $\varphi:\left\{t_{l}, t_{r}\right\}^{+} \rightarrow\left[T_{l}, T_{r}\right]$ be the epimorphism defined by $t_{l} \varphi=T_{l}, t_{r} \varphi=T_{r}$. Let $\rho=\varphi \circ \varphi^{-1}$ and $\sigma$ be the congruence on $\left\{t_{l}, t_{r}\right\}^{+}$defined by the relation $\left\{\left(t_{l}^{2}, t_{l}\right),\left(t_{r}^{2}, t_{r}\right)\right\}$. Since $T_{l}^{2}=T_{l}$ and $T_{r}^{2}=T_{r}$, it follows that $\sigma \subseteq \rho$. To establish the reverse inclusion, let $p, q \in\left\{t_{l}, t_{r}\right\}^{+}$and $p \rho q$. Using the relation defining $\sigma$, there exist $v, w \in$ $\Delta_{t_{l} t_{r}}$ such that $p \sigma v$ and $q \sigma w$. Since $\sigma \subseteq \rho$, we have $v \rho w$. Thus $v\left(T_{l}, T_{r}\right)=$ $v \varphi=w \varphi=w\left(T_{l}, T_{r}\right)$. Now let $T_{l}{ }^{*}, T_{r}{ }^{*}$ be the operators defined on $\mathscr{L}(\mathscr{B})$ by

$$
\mathscr{U} T_{1}^{*}=\mathscr{L} \mathscr{Z} \circ \mathscr{U}, \quad \mathscr{U} T_{r}^{*}=\mathscr{R} \mathscr{Z} \circ \mathscr{U} .
$$

By repeated application of Lemma 7.6, we have that for all $\mathscr{U} \in \mathscr{L}(\mathscr{B})$,

$$
\begin{aligned}
\mathscr{U} v\left(T_{l}^{*}, T_{r}^{*}\right) & =\mathscr{U} v\left(T_{l}, T_{r}\right) \cap \mathscr{B} \\
& =\mathscr{U} w\left(T_{l}, T_{r}\right) \cap \mathscr{B}=\mathscr{U} w\left(T_{l}^{*}, T_{r}^{*}\right) .
\end{aligned}
$$

By $([8]$, Example 10), this implies that $v=w$. Thus $p \sigma v=w \sigma q$ so that $p \sigma q$ and $\sigma \subseteq \rho$. Thus $\sigma=\rho$ and (iv) holds.

Parts (v) and (vi) follow directly from Lemma 7.7. 
8. The semigroup generated by $C, L, K$ and $T$. In $\S \S 3$ and 4 we have considered the semigroups $[C, L]$ and $[K, T]$ and in $\S \S 5$ and 6 we have shown that the operators $C$ and $L$ commute with the operators $K, T, T_{l}$ and $T_{r}$. In this section we will show that the relations between $C, L, K$ and $T$ already introduced are sufficient to describe $[C, L, K, T]$.

We require three technical preliminary lemmas.

Lemma 8.1. Let $\mathscr{U}, \mathscr{V} \in \mathscr{L}(\mathscr{C} \mathscr{R}), \mathscr{S} \subseteq \mathscr{U}, \mathscr{N} \mathscr{B} \subseteq \mathscr{V}, \mathscr{U} \subset \mathscr{V}$ and $w \in$ $\Delta_{k t}$. If either (i) $\mathscr{V} T=\mathscr{V}$ and $w=k w_{1}$ for some $w_{1} \in \Delta_{k t} \cup\{\varnothing\}$ or (ii) $\mathscr{V} K=\mathscr{V}$ and $w=t w_{1}$ for some $w_{1} \in \Delta_{k t} \cup\{\varnothing\}$, then $\mathscr{U} w(K, T) \subset$ $\mathscr{V} w(K, T)$.

Proof. The assumption that $\mathscr{U} \subseteq \mathscr{V}$ implies that $\mathscr{U} w(K, T) \subseteq$ $\mathscr{V} w(K, T)$ for all $w \in \Delta_{k t}$. So the objective is to show that under certain circumstances, inequality is preserved. We will only consider case (i), since case (ii) will follow in a similar manner using Lemma 7.3. We will argue by induction on the length of $w_{1}$.

First suppose that $w_{1}=\varnothing$. Since $\mathscr{V} \nsubseteq \mathscr{U}$, it follows from Lemma 7.2 that $\mathscr{V} T \nsubseteq \mathscr{U} K$ so that $\mathscr{V}=\mathscr{V} T \nsubseteq \mathscr{U} K$ and therefore

$$
\mathscr{V} w(K, T)=\mathscr{V} K \nsubseteq \mathscr{U} K=\mathscr{U} w(K, T) .
$$

Now suppose that $\left|w_{1}\right| \geq 1$ and that the desired inequality holds for any words of the form $k w_{1}^{\prime} \in \Delta_{k t}$, where $\left|w_{1}^{\prime}\right|<\left|w_{1}\right|$. We have two cases.

Case 1. $w_{1}=w_{2} k$ for some $w_{2} \in \Delta_{k t} \cup\{\varnothing\}$. In fact, we must have $w_{2} \neq \varnothing$ since otherwise we obtain $w=k w_{1}=k w_{2} k=k \varnothing k=k k$ which is not distinguished. Thus $\left|w_{2}\right| \geq 1$ and, since $w_{1}=w_{2} k, w_{2}$ must end in $t$. Hence $w_{2}(K, T) T=w_{2}(K, T)$. By the induction hypothesis, $\mathscr{U} K w_{2}(K, T)$ $\subset \mathscr{V} K w_{2}(K, T)$. In particular, $\mathscr{V} K w_{2}(K, T) \nsubseteq \mathscr{U} K w_{2}(K, T)$. Therefore, by Lemma 7.2,

$$
\mathscr{V} K w_{2}(K, T) T \nsubseteq \mathscr{U} K w_{2}(K, T) K=\mathscr{U} w(K, T)
$$

so that

$$
\mathscr{V} K w_{2}(K, T)=\mathscr{V} K w_{2}(K, T) T \nsubseteq \mathscr{U} w(K, T)
$$

and

$$
\mathscr{V} w(K, T)=\mathscr{V} K w_{2}(K, T) K \nsubseteq \mathscr{U} w(K, T) .
$$

Case 2. $w_{1}=w_{2} t$ for some $w_{2} \in \Delta_{k t} \cup\{\varnothing\}$. The proof in this case is similar to that of Case 1 .

LEMmA 8.2. Let $r, s \in \Delta_{c l}$ and $v, w \in \Delta_{k t} \cup\{\varnothing\}$ be such that $r(C, L) v(K, T)=s(C, L) w(K, T)$. Then $v=w$. 
Proof. Let $R=r(C, L), S=s(C, L), V=v(K, T), W=w(K, T)$ and $\mathscr{U}=\mathcal{O} \mathscr{N} \mathscr{B} \mathscr{G}$. Then $\mathscr{U} C=\mathscr{U} L=\mathscr{U}$ so that $\mathscr{U} R=\mathscr{U} S=\mathscr{U}$. Hence $\mathscr{U} V=\mathscr{U} W$. By Lemma 7.4, either (i) $v=w$, as claimed, or (ii) $|v|=|w|+1$ and $v$ and $w$ end in the same letter. So let (ii) hold. Since occurrences of $k$ and $t$ alternate in $v$ and $w$ and they end in the same letter, we must have $v=k w$ or $v=t w$.

Let $v=k w$. If $w=\varnothing$, then $\mathscr{U} V=\mathscr{U} K=\mathcal{O} \neq \mathcal{O} \mathscr{N} \mathscr{B} \mathscr{G}=\mathscr{U} W$, a contradiction. Hence $w \neq \varnothing$ and, since $v$ is distinguished, $w$ must begin with $t$. Let $\mathscr{V}=\mathscr{U} K=\mathcal{O}$. Then $\mathscr{V} K=\mathscr{V}, \mathscr{U} \subset \mathscr{V}$ and $w=t w_{1}$ for some $w_{1} \in \Delta_{k t} \cup\{\varnothing\}$. By Lemma 8.1(ii),

$$
\mathscr{U} w(K, T) \subset \mathscr{V} w(K, T)=\mathscr{U} K w(K, T)=\mathscr{U} v(K, T)
$$

which is a contradiction. Thus $v=w$.

The case when $v=t w$ is treated similarly.

Lemma 8.3. For distinct $A, B \in\{\varnothing, C, L, C L, L C\}$, there exist $\mathscr{U}=$ $\mathscr{U}_{A, B}, \mathscr{V}=\mathscr{V}_{A, B} \in \mathscr{L}(\mathscr{C} \mathscr{R})$ such that

(i) $\mathscr{N} \mathscr{B} \subseteq \mathscr{U} \cap \mathscr{V}$,

(ii) for $\mathscr{X} \in\{\mathscr{U}, \mathscr{V}\}$, either $\mathscr{X} A \subset \mathscr{X} B$ or $\mathscr{X} B \subset \mathscr{X} A$,

(iii) $\mathscr{U} A K=\mathscr{U} A, \mathscr{U} B K=\mathscr{U} B$,

(iv) $\mathscr{V} A T=\mathscr{V} A, \mathscr{V} B T=\mathscr{V} B$.

Proof. For all choices of distinct elements $A, B, \in\{\varnothing, C, L, C L, L C\}$, we display in the table below suitable choices for $\mathscr{U}$. The square in the row labelled with $A$ and the column labelled with $B$ has the form

$$
\begin{array}{|l|l|}
\hline \multicolumn{2}{|c|}{\text { where } \mathscr{P}=\mathscr{U} A \text { and } \mathscr{Q}=\mathscr{U} B .} \\
\hline \mathscr{P} & \mathscr{Q} \\
\hline
\end{array}
$$

In all cases $\mathscr{U}$ is either $\mathscr{B}$ or $\mathcal{O}$ so that $\mathscr{U}$ satisfies the requirement (i). In addition, in all cases either $\mathscr{P} \subset \mathscr{Q}$ or $\mathscr{Q} \subset \mathscr{P}$ so that $\mathscr{U}$ also satisfies the

\begin{tabular}{|c|c|c|c|c|c|}
\hline$A-B$ & \multicolumn{2}{|c|}{$C$} & $L$ & $C L$ & $L C$ \\
\hline \multirow{2}{*}{$\varnothing$} & \multicolumn{2}{|c|}{$\mathscr{B}$} & 0 & $\mathscr{B}$ & $\mathscr{B}$ \\
\hline & \begin{tabular}{l|l|}
$\mathscr{B}$ \\
\end{tabular} & 0 & \begin{tabular}{l|l}
$\mathcal{O}$ & $\mathcal{O} L$ \\
\end{tabular} & \begin{tabular}{l|l}
$\mathscr{B}$ & $\mathcal{O} L$ \\
\end{tabular} & \begin{tabular}{l|l}
$\mathscr{B}$ & $\mathcal{O}$ \\
\end{tabular} \\
\hline \multirow{2}{*}{$C$} & & & $\mathscr{B}$ & $\mathscr{B}$ & 0 \\
\hline & & & \begin{tabular}{l|l}
$\mathcal{O}$ & $\mathscr{B}$ \\
\end{tabular} & \begin{tabular}{l|l}
$\mathcal{O}$ & $\mathcal{O} L$ \\
\end{tabular} & \begin{tabular}{l|l|}
$\mathcal{O}$ & $\mathcal{O} L$ \\
\end{tabular} \\
\hline \multirow{2}{*}{$L$} & & & & $\mathscr{B}$ & $\mathscr{B}$ \\
\hline & & & & \begin{tabular}{l|l}
$\mathscr{B}$ & $\mathcal{O} L$ \\
\end{tabular} & \begin{tabular}{l|l}
$\mathscr{B}$ & $\mathcal{O}$ \\
\end{tabular} \\
\hline \multirow{2}{*}{$C L$} & & & & & $\mathscr{B}$ \\
\hline & & & & & \begin{tabular}{l|l}
$O L$ & $\mathcal{O}$
\end{tabular} \\
\hline
\end{tabular}
requirement (ii). 
By Lemmas 5.3 and 6.3, $K$ commutes with $C$ and $L$ and therefore also with $A$ and $B$. It is easily seen that $\mathscr{B} K=\mathscr{B}$ and $\mathcal{O} K=\mathscr{O}$. Consequently, since in all cases $\mathscr{U}$ is either $\mathscr{B}$ or $\mathcal{O}$, we have that $\mathscr{U} K=\mathscr{U}$. Hence

$$
\mathscr{U} A K=\mathscr{U} K A=\mathscr{U} A, \quad \mathscr{U} B K=\mathscr{U} K B=\mathscr{U} B
$$

and (iii) holds.

For each choice of $A$ and $B$, we now take $\mathscr{V}=\mathscr{U T}$. Then $\mathscr{N} \mathscr{B} \subseteq \mathscr{U}$ $\subseteq \mathscr{U} T=\mathscr{V}$ so that $\mathscr{V}$ satisfies (i). By (ii), either $\mathscr{U} A \subset \mathscr{U} B$ or $\mathscr{U} B \subset \mathscr{U} A$. First suppose that $\mathscr{U} A \subset \mathscr{U} B$. By (iii) we have that $(\mathscr{U} A) K=\mathscr{U} A$ and $(\mathscr{U} B) K=\mathscr{U} B$ and hence, by Lemma 8.1(ii), $\mathscr{U} A T \subset \mathscr{U} B T$. By Lemmas 5.5 and 6.5, $T$ commutes with $C$ and $L$ and therefore with $A$ and $B$. Hence

$$
\mathscr{V} A=\mathscr{U} T A=\mathscr{U} A T \subset \mathscr{U} B T=\mathscr{U} T B=\mathscr{V} B .
$$

Similarly, if $\mathscr{U} B \subset \mathscr{U} A$ we obtain $\mathscr{V} B \subset \mathscr{V} A$ so that $\mathscr{V}$ satisfies (ii). Finally, again since $T$ commutes with $A$ and $B$ and also since $T^{2}=T$,

$$
\begin{aligned}
& \mathscr{V} A T=\mathscr{V} T A=\mathscr{U} T T A=\mathscr{U} T A=\mathscr{V} A, \\
& \mathscr{V} B T=\mathscr{V} T B=\mathscr{U} T T B=\mathscr{U} T B=\mathscr{V} B .
\end{aligned}
$$

Thus $\mathscr{V}$ satisfies (iv).

LEMMA 8.4. Let $w \in \Delta_{k t} \cup\{\varnothing\}$ and $W=w(K, T)$. Then the following are distinct: $W, C W, L W, C L W, L C W$.

Proof. If $w=\varnothing$, then the result follows from the description of the semigroup $[C, L]$ in $\S 3$. Now let $A, B \in\{\varnothing, C, L, C L, L C\}$ and suppose first that $w$ begins with the letter $t$. By Lemma 8.3, there exists a variety $\mathscr{U}=\mathscr{U}_{A, B}$, satisfying Lemma 8.3(i)-(iii). By Lemma 8.1(ii), we must have $\mathscr{U} A W \neq \mathscr{U} B W$ so that $A W \neq B W$. Suppose, on the other hand, that $w$ begins with the letter $k$. Let $\mathscr{V}=\mathscr{V}_{A, B} \in \mathscr{L}(\mathscr{C} \mathscr{R})$ satisfy Lemma 8.3(i), (ii) and (iv). By Lemma 8.1(i), we must have $\mathscr{V} A W \neq \mathscr{V} B W$ so that again $A W \neq B W$.

We now apply these observations to the semigroup $[C, L, K, T]$.

THEOREM 8.5.

$$
\begin{array}{r}
{[C, L, K, T] \cong\langle c, l, k, t| c^{2}=c, l^{2}=l, k^{2}=k,} \\
t^{2}=t, c l c=l c l=c l, c k=k c, \\
c t=t c, l k=k l, l t=t l\rangle .
\end{array}
$$


Proof. Let $N$ be the semigroup on the right hand side of the statement of the theorem. Let $M=\{c, l, k, t\}^{+}$and $\varphi: M \rightarrow[C, L, K, T]$ be the epimorphism defined by $c \varphi=C, l \varphi=L, k \varphi=K, t \varphi=T$. Let $\rho=\varphi \circ \varphi^{-1}$ and $\sigma$ be the congruence on $M$ generated by the defining relations for $N$.

It follows from the fact that the operators $C, L, K$ and $T$ are all idempotent and from Lemmas 3.1, 3.3, 5.3, 5.5, 6.3 and 6.5 that $\sigma \subseteq \rho$. Now suppose that $p \rho q$. Using the relations defining $\sigma$, there exist $r, s \in \Delta_{c l}$ and $v, w \in \Delta_{k t}$ such that $p \sigma r v$ and $q \sigma s w$. Since $\sigma \subseteq \rho$, this implies that $r v \rho s w$. Hence $(r v) \varphi=(s w) \varphi$ so that $r(C, L) v(K, T)=$ $s(C, L) w(K, T)$. By Lemma 8.2, $v=w$ and, by Lemma 8.4, $r=s$. Thus $p \sigma r v=s w \sigma q$. Hence $\rho \subseteq \sigma$, as required.

Corollary 8.6. $[C, L, K, T]^{1} \cong[C, L]^{1} \times[K, T]^{1}$.

Proof. This is immediate from Theorem 8.5.

Similar descriptions to that of $[C, L, K, T]$ in Theorem 8.5 can be obtained for [ $\left.C, L, K, T_{l}\right],\left[C, L, K, T_{r}\right]$ and $\left[C, L, T_{l}, T_{r}\right.$ ] by substituting $T_{l}$ for $T$ etc. in all arguments. The nature of the semigroup generated by $C, L, K, T, T_{l}$ and $T_{r}$ is left open.

9. Evaluation at the trivial variety. The diagram below presents a few varieties obtained by repeated application of the operators $C, L, K, T, T_{l}$ and $T_{r}$ to the trivial variety $\mathscr{T}$. In order to save writing $\mathscr{T}$ in the expressions of the form $\mathscr{T} C, \mathscr{T} T, \mathscr{T} C T_{r}$, etc., we have written only $C, T, C T_{r}$, etc. For example, the vertex labelled $\mathscr{G}=C=T$ stands for the variety of groups; this variety can also be described as $\mathscr{T C}$ or $\mathscr{T} T$. The following legend is used to denote the classes induced by these operators:

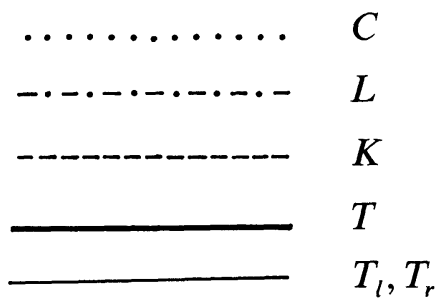

We omit the proofs of the statements implicit in the diagram. 


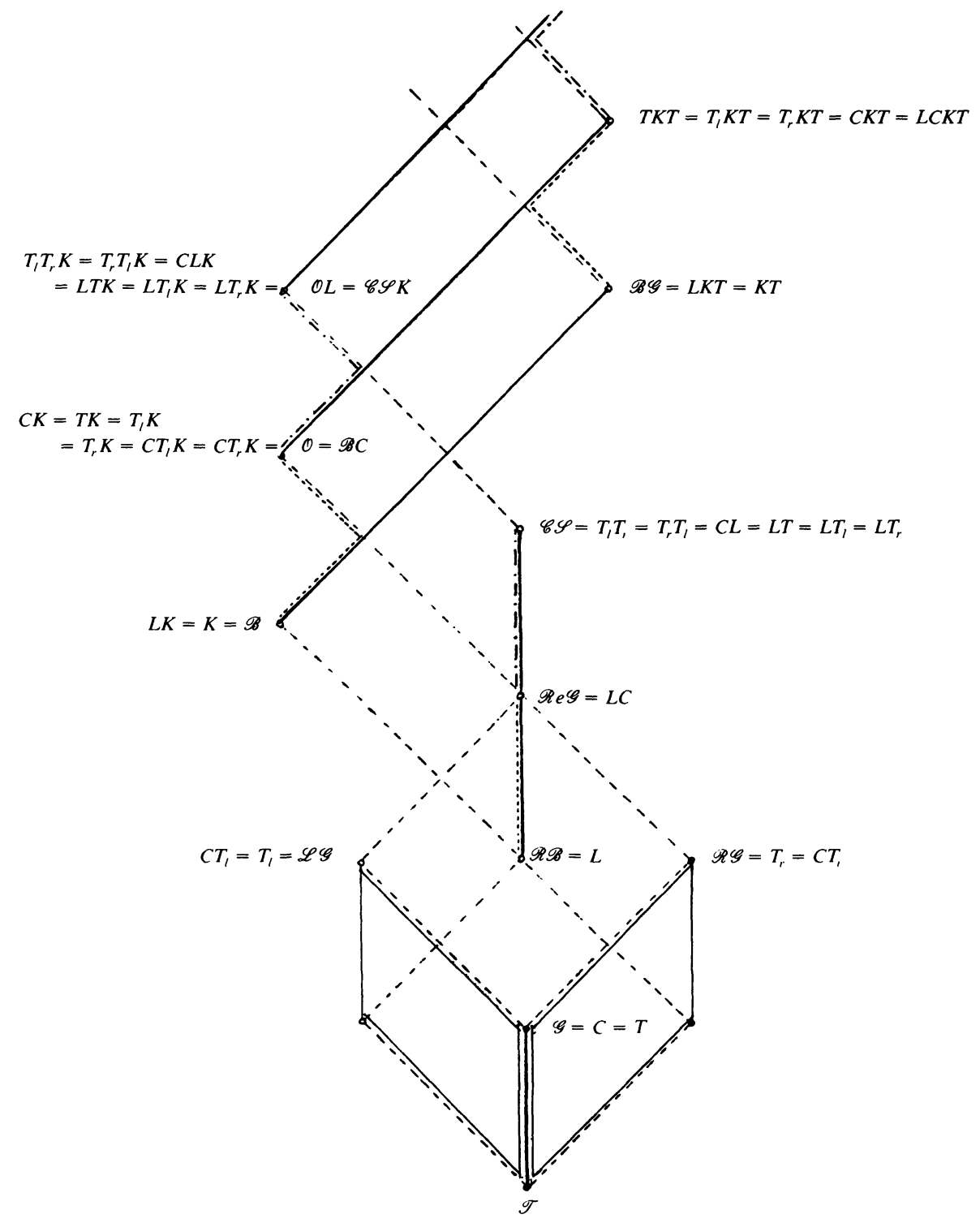

Acknowledgment. The authors are indebted to J. Kadourek for detecting an error in an earlier version of the paper.

\section{REFERENCES}

[1] R. Feigenbaum, Regular semigroup congruences, Semigroup Forum 17 (1979), 371-377.

[2] J. A. Gerhard, Free completely regular semigroups II: Word Problem, J. Algebra, 82 (1983), 143-156. 
[3] T. E. Hall, On regular semigroups, J. Algebra, 24 (1973), 1-24.

[4] T. E. Hall and P. R. Jones, On the lattice of varieties of bands of groups, Pacific J. Math., 91 (1980), 327-337.

[5] J. M. Howie, An Introduction to Semigroup Theory, Academic Press, London, 1976.

[6] P. R. Jones, On the lattice of varieties of completely regular semigroups, J. Austral. Math. Soc., A35 (1983), 227-235.

[7] , Mal'cev products of varieties of completely regular semigroups, (preprint).

[8] J. Kadourek, On the word problem for free bands of groups and for free objects in some other varieties of completely regular semigroups, Semigroup Forum, (to appear).

[9] F. Pastijn and M. Petrich, The congruence lattice of a regular semigroup, (preprint).

[10] F. J. Pastijn and P. G. Trotter, Lattices of completely regular semigroups, Pacific J. Math., 119 (1985), 191-214.

[11] M. Petrich, Introduction to Semigroups, Merrill, Columbus, 1973.

[12] M. Petrich and N. R. Reilly, Varieties of groups and of completely simple semigroups, Bull. Austral. Math. Soc., 23 (1981), 339-359.

[13] N. R. Reilly, Varieties of completely regular semigroups. J. Austral. Math. Soc., A38 (1985), 372-393.

Received July 10, 1986 and in revised form December 12, 1986. Research by the second author was supported, in part, by NSERC grant A4044.

SimON Fraser UNIVERSITY

Burnaby, B. C. CANADA

V5A 1S6 



\section{PACIFIC JOURNAL OF MATHEMATICS \\ EDITORS}

V. S. VARADARAJAN

(Managing Editor)

University of California

Los Angeles, CA 90024

Herbert Clemens

University of Utah

Salt Lake City, UT 84112

R. FINN

Stanford University

Stanford, CA 94305
HERMANN FLASCHKA

University of Arizona

Tucson, AZ 85721

RAMESH A. GANGOLLI

University of Washington Seattle, WA 98195

VAUGHAN F. R. JONES

University of California

Berkeley, CA 94720
ROBION KIRBY

University of California

Berkeley, CA 94720

C. C. MOORE

University of California

Berkeley, CA 94720

HAROLD STARK

University of California, San Diego

La Jolla, CA 92093

\section{ASSOCIATE EDITORS}
R. ARENS
E. F. BECKENBACH
B. H. NEUMANN
F. WOLF
K. YOSHIDA
(1906-1982)

\section{SUPPORTING INSTITUTIONS}

UNIVERSITY OF ARIZONA

UNIVERSITY OF BRITISH COLUMBIA

CALIFORNIA INSTITUTE OF TECHNOLOGY

UNIVERSITY OF CALIFORNIA

MONTANA STATE UNIVERSITY

UNIVERSITY OF NEVADA, RENO

NEW MEXICO STATE UNIVERSITY

OREGON STATE UNIVERSITY
UNIVERSITY OF OREGON UNIVERSITY OF SOUTHERN CALIFORNIA

STANFORD UNIVERSITY

UNIVERSITY OF HAWAII

UNIVERSITY OF TOKYO

UNIVERSITY OF UTAH

WASHINGTON STATE UNIVERSITY

UNIVERSITY OF WASHINGTON 


\section{Pacific Journal of Mathematics \\ Vol. 132, No. $1 \quad$ January, 1988}

Scott W. Brown, Full analytic subspaces for contractions with rich spectrum ...1

Robert Main Burton, Jr. and Tae-Sung Kim, An invariance principle for

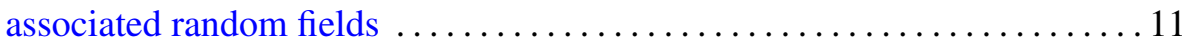

Ana M. Viola-Prioli and Jorge Viola-Prioli, Rings whose kernel functors

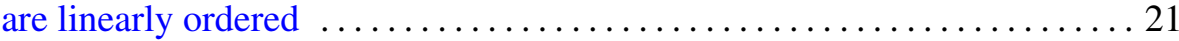

David E. Handelman, Representing polynomials by positive linear

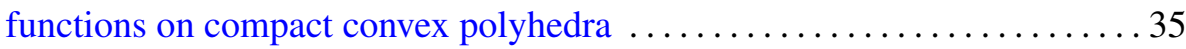

Patrick Keef, On the Tor functor and some classes of abelian groups . .....66

Dennis R. Malm, Simplicity of partial and Schmidt differential operator

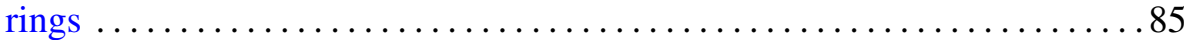

José M. Montesinos and Carmen Safont, On the Birman invariants of

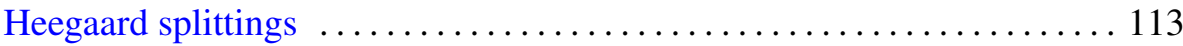

N. P. Mukherjee and Prabir Bhattacharya, The normal index of a finite group

Mario Petrich and Norman R. Reilly, Semigroups generated by certain operators on varieties of completely regular semigroups

Robert Tijdeman and Lian Xiang Wang, Sums of products of powers of given prime numbers

Joel Larry Weiner, First integrals for a direction field on a simply connected plane domain 